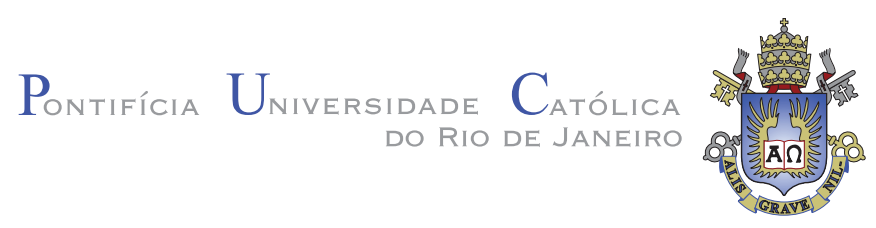

Edward José Pacheco Condori

\title{
Deployment of Distributed Component-based Applications on Cloud Infrastructures
}

\section{DISSERTAÇÃO DE MESTRADO}

Dissertation presented to the Programa de Pós-Graduação em Informática of the Departmento de Informática, PUC-Rio as partial fulfillment of the requirements for the degree of Mestre em Informática.

Advisor: Prof. Renato Fontoura de Gusmão Cerqueira

Rio de Janeiro

September 2012 


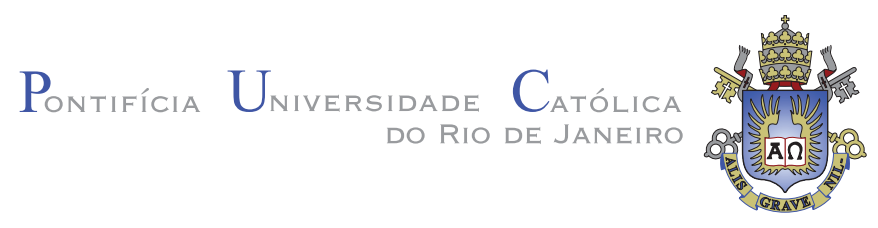

Edward José Pacheco Condori

\section{Deployment of Distributed Component-based Applications on Cloud Infrastructures}

Dissertation presented to the Programa de Pós-Graduação em Informática of the Departmento de Informática do Centro Técnico Científico da PUC-Rio, as partial fulfillment of the requirements for the degree of Mestre.

Prof. Renato Fontoura de Gusmão Cerqueira

Advisor

Departmento de Informática — PUC-Rio

Prof. Noemi de La Rocque Rodríguez

Departmento de Informática - PUC-Rio

Prof. Antônio Tadeu Azevedo Gomes

LNCC

Prof. José Eugenio Leal

Coordinator of the Centro Técnico Científico - PUC-Rio

Rio de Janeiro — September 14, 2012 
All rights reserved.

\section{Edward José Pacheco Condori}

Edward Pacheco graduated from the Universidad Nacional de San Agustin (Arequipa, Perú) in Ingenieria de Sistemas.

Ficha Catalográfica

Pacheco Condori, Edward José

Deployment of distributed component-based applications on cloud infrastructures / Edward José Pacheco Condori; adviser: Renato Fontoura de Gusmão Cerqueira - 2012.

91 f.: il. (color.) ; $30 \mathrm{~cm}$

1. Dissertação (mestrado) - Pontifícia Universidade Católica do Rio de Janeiro, Departmento de Informática, 2012.

Inclui bibliografia

1. Informática - Teses. 2. Sistema de componentes de software (SCS). 3. Aplicações distribuídas. 4. Infraestrutura de implantação. 5. Infraestrutura na nuvem. 6. Cloud API. I. Cerqueira, Renato Fontoura de Gusmão. II. Pontifícia Universidade Católica do Rio de Janeiro. Departmento de Informática. III. Título. 


\section{Acknowledgments}

To my mother, father, sisters, and brother, and all my family for their unconditional love. To my advisor, prof. Renato Cerqueira, who offered me the opportunity to be part of the Distributed System Group. To the CAPES and Tecgraf, for the financial support, without which this work would not have been realized. To all my colleagues of the PUC-Rio for the continued support. 


\section{Abstract}

Pacheco Condori, Edward José; Cerqueira, Renato Fontoura de Gusmão. Deployment of Distributed Component-based Applications on Cloud Infrastructures. Rio de Janeiro, 2012. 91p. MSc. Dissertation — Departmento de Informática, Pontifícia Universidade Católica do Rio de Janeiro.

Deployment of distributed component-based applications is composed of a set of activities managed by a Deployment Infrastructure. Current applications are becoming increasingly more complex, requiring a multi-platform and a dynamic target environment. Thus, the planning activity is the most critical step because it defines the configuration of the execution infrastructure in order to satisfy the requirements of the application's target environment. On the other hand, the cloud service model called "Infrastructure as a Service" (IaaS) offers on-demand computational resources with dynamic, scalable, and elastic features. In this work we have extended the Deployment Infrastructure for SCS components to support private or public clouds as its target environment, through the use of a cloud API and flexible policies to specify a customized target environment. Additionally, we host the Deployment Infrastructure on the cloud, which allow us to use on-demand computational resources to instantiate Deployment Infrastructure services, creating an experimental "Platform as a Service" (PaaS).

\section{Keywords}

Sofware Component System(SCS). Distributed Applications. Deployment Infrastructure. Cloud Infrastructures. Cloud API. 


\section{Resumo}

Pacheco Condori, Edward José; Cerqueira, Renato Fontoura de Gusmão. Implantação de Aplicações baseadas em Componentes Distribuídos sobre Infraestruturas na Nuvem. Rio de Janeiro, 2012. 91p. Dissertação de Mestrado Departamento de Informática, Pontifícia Universidade Católica do Rio de Janeiro.

A implantação de aplicações baseadas em componentes distribuídos é composta por um conjunto de atividades geridas por uma Infraestrutura de Implantação. Aplicações atuais estão se tornando cada vez mais complexas, necessitando de um ambiente alvo dinâmico e multi-plataforma. Assim, a atividade de planejamento de uma implantação é o passo mais crítico, pois define a configuração da infraestrutura de execução de forma a atender os requisitos do ambiente alvo de uma aplicação. Por outro lado, o modelo de serviço na nuvem chamado "Infraestrutura como Serviço(IaaS)" oferece recursos computacionais sob demanda, com características dinâmicas, escaláveis e elásticas. Nesta dissertação nós estendemos a Infraestrutura de Implantação para componentes SCS de forma a permitir o uso de nuvens privadas ou públicas como o ambiente alvo de uma implantação, através do uso de uma cloud API e políticas flexíveis para especificar um ambiente alvo personalizado. Além disso, hospedamos a infraestrutura de implantação na nuvem. Isto permitiu-nos usar recursos computacionais sob demanda para

instanciar os serviços da Infraestrutura de Implantação, produzindo uma "Plataforma como Serviço(PaaS)" experimental.

\section{Palavras-chave}

Sistema de Componentes de Software(SCS). Aplicações Distribuídas. Infraestrutura de Implantação. Infraestruturas na Nuvem. Cloud API. 


\section{Contents}

1 Introduction $\quad 11$

1.1 Proposal . . . . . . . . . . . . . . . . . . . . 13

1.2 Objectives . . . . . . . . . . . . . . . . . . 14

1.3 Contributions . . . . . . . . . . . . . . . . . . . . 14

1.4 Dissertation Structure . . . . . . . . . . . . . . . . . 15

2 Background Work 16

2.1 Cloud Computing Fundamentals . . . . . . . . . . . . . 16

2.1.1 What is Cloud Computing? . . . . . . . . . . . . . 16

2.1.2 Cloud Taxonomy . . . . . . . . . . . . . . . . . 18

2.1.3 Cloud Platforms . . . . . . . . . . . . . . . 20

2.1.4 Cloud APIs . . . . . . . . . . . . . . . . . 22

2.1.5 Cloud Software Stack . . . . . . . . . . . . . . . . 23

2.2 Related Work . . . . . . . . . . . . . . . . . . 25

2.2.1 Deployment Process . . . . . . . . . . . . . . . 25

2.2.2 Deployment on Grid Environments . . . . . . . . . . . . . 27

Fractal and DeployWare . . . . . . . . . . . . . 27

ADAGE and CoRDAGe . . . . . . . . . . . . . 29

$\mathrm{DAnCE} \ldots \ldots \ldots \ldots \ldots \ldots$

2.2.3 Deployment on Cloud Infrastructures . . . . . . . . . . 32

FraSCAti . . . . . . . . . . . . . . . . 32

OSGi .......................... 34

2.3 Final Remarks . . . . . . . . . . . . . . . . . 35

3 Reference Scenario $\quad 37$

3.1 SCS Component Model . . . . . . . . . . . . . . 37

3.2 Execution Infrastructure . . . . . . . . . . . . . . . . . . . . . . 38

3.3 Deployment Infrastructure . . . . . . . . . . . . . . . . . . . 39

3.3.1 Deployment Planning . . . . . . . . . . . . . . . . . . . 40

4 SCS Deployment Infrastructure on Cloud Infrastructures 42

4.1 Challenges . . . . . . . . . . . . . . . . . . . . . . . 42

4.1 .1 Target Environment Requirements . . . . . . . . . . 44

4.1.2 Cloud Infrastructure API . . . . . . . . . . . . 44

4.1 .3 User Management . . . . . . . . . . . . . . . . 44

4.1.4 Setting up a Cloud Computing Environment . . . . . . . . . . 45

4.2 Cloud Resource Provisioning . . . . . . . . . . . . . . . . 45

4.2.1 A Cloud Computing Environment . . . . . . . . . . . 46

Setting up a private cloud . . . . . . . . . . . . 46

Accessing a public cloud . . . . . . . . . . . . . . . . . . . . 48

4.2 .2 Cloud API. . . . . . . . . . . . . . . 48 


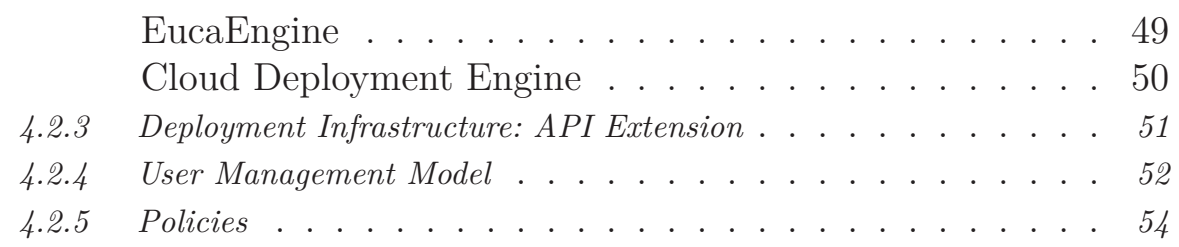

4.3 Configuring SCS Deployment Infrastructure on the Cloud . . . 55

4.3.1 Architectural Overview . . . . . . . . . . . . . . . . . . 56

4.3.2 Deployment Infrastructure: PaaS . . . . . . . . . . . . . . . . 59

Minimum Supported Configuration . . . . . . . . . 60

A Multi-Deployment Configuration . . . . . . . . . 60

4.3.3 Deployment Infrastructure: IaaS . . . . . . . . . . . . . . . . 61

4.3.4 Prototype and Limitations . . . . . . . . . . . . . . . . . . . 62

5 SCS Deployment Infrastructure in Use $\quad 66$

5.1 MapReduce Application . . . . . . . . . . . . . . . . 66

5.2 Deploying a SCS-MapReduce on the Cloud . . . . . . . . . 68

5.3 Final Considerations . . . . . . . . . . . . . . . 70

6 Conclusions and Future Work $\quad 74$

$\begin{array}{ll}\text { Bibliography } & 76\end{array}$

A Appendix $\quad 82$

A.1 Cloud Infrastructure Service IDL . . . . . . . . . . . . . . 82

A.2 SCS MapdReduce - Deployment Script . . . . . . . . . . . . . 82

A.3 SCS MapdReduce on Cloud Infrastructures - Deployment Script 82 


\section{List of Figures}

2.1 Conceptual Reference Model . . . . . . . . . . . . . . . . . . . 19

2.2 A Generic Deployment Process of distributed

Component-based Applications . . . . . . . . . . . . . 27

2.3 Representing physical resources using a physical tree . . . . . . 31

2.4 Internal Architecture and Deployment Stages of DAnCE . . . 32

$3.1 \quad$ SCS Component Model . . . . . . . . . . . . . . . . 38

3.2 SCS Execution Infrastructure . . . . . . . . . . . . . . 39

3.3 Overview of SCS Deployment Infrastructure . . . . . . . . . . 40

4.1 A Generic Deployment Process of distributed Component-based Applications on Cloud Infrastructures . . . 43

4.2 OpenStack Cloud Platform . . . . . . . . . . . . . . . . . . 47

4.3 Cloud API - Architecture . . . . . . . . . . . . . . . . . 49

4.4 EucaEngine - Instance Class . . . . . . . . . . . . . . . 50

4.5 Cloud Engine . . . . . . . . . . . . . . . . . . . . . 52

4.6 DeployManager Extensions . . . . . . . . . . . . . 53

4.7 User Management . . . . . . . . . . . . . . . . . . . . . . 54

4.8 Deployment Infrastructure Architectural Overview . . . . . . . 57

4.9 Deployment Infrastructure on Cloud Infrastructures: Minimal Architecture . . . . . . . . . . . . . . 6 60

4.10 A Multi-Deployment Architectural Configuration . . . . . . . . 61

5.1 MapReduce Architecture . . . . . . . . . . . . . . . . . 67

5.2 A SCS Component-based MapReduce Framework Architecture 68

5.3 Deploying a SCS-based MapReduce Application on a Cloud Infrastructure . . . . . . . . . . . . . . . . . . . . . . 69 


\section{List of Tables}

4.1 Type of Users for Deployment Infrastructure on Cloud
Infrastructures . . . . . . . . . . . . . . . . . . . 53 . 54 . . . 54 Policies required to specify the deployment of applications. . .

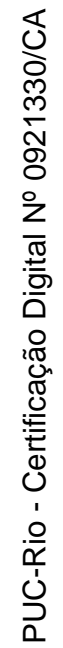

